\title{
Parafermionic quasi-particle basis and fermionic-type characters
}

\author{
P. Jacob and P. Mathieu \\ Département de physique, \\ Université Laval, \\ Québec, Canada G1K 7P4 \\ (pjacob@phy.ulaval.ca, pmathieu@phy.ulaval.ca)
}

\begin{abstract}
A new basis of states for highest-weight modules in $\mathbb{Z}_{k}$ parafermionic conformal theories is displayed. It is formulated in terms of an effective exclusion principle constraining strings of $k$ fundamental parafermionic modes. The states of a module are then built by a simple filling process, with no singular-vector subtractions. That results in fermionicsum representations of the characters, which are exactly the Lepowsky-Primc expressions. We also stress that the underlying combinatorics - which is the one pertaining to the Andrews-Gordon identities - has a remarkably natural parafermionic interpretation.
\end{abstract}

06/01 


\section{Introduction}

\subsection{Bosonic- vs fermionic-sum representation of characters}

Over the last years, there has been a great amount of activities centered on novel i.e., fermionic-like - representations of the characters in rational conformal field theories. The standard expression for the irreducible characters is obtained by the subtraction of the singular vectors in reducible Verma modules (which is usually an infinite sequence of subtractions and additions). The prototype of such character expressions are the wellknown Rocha-Caridi formulae for irreducible highest-weight modules $\left|h_{r s}\right\rangle, h_{r s}=[(p r-$ $\left.\left.p^{\prime} s\right)^{2}-\left(p-p^{\prime}\right)^{2}\right] / 4 p p^{\prime}$, in Virasoro minimal models [1]:

$$
\chi_{r s}(q)=\frac{q^{-1 / 24}}{(q)_{\infty}} \sum_{n \in \mathbb{Z}}\left[q^{\left(2 p p^{\prime} n+p r-p^{\prime} s\right)^{2} / 4 p p^{\prime}}-q^{\left(2 p p^{\prime} n+p r+p^{\prime} s\right)^{2} / 4 p p^{\prime}}\right]
$$

We used the following notation for the Euler function:

$$
(q)_{\infty}=\lim _{n \rightarrow \infty}(q)_{n}, \quad(q)_{n}=\prod_{i=1}^{n}\left(1-q^{i}\right)
$$

with $(q)_{0}=1$. The Kac-Peterson characters of the integrable representations in affine Lie algebras provide the analogue for WZW models [2].

Characters of that type are termed 'bosonic' in [3] (see also [4]) since they can be obtained by a BRST projection of free-field (typically, bosonic) representations (cf. [5] for the minimal models). More precisely, such characters are obtained by the free application of the various lowering operators of the underlying infinite algebra and the subsequent corrections resulting from the subtraction of the singular vectors. For the Virasoro case, the ladder operators are the $L_{n<0}$ modes and their free action is signaled in (1.1) by the presence of the factor $(q)_{\infty}^{-1}$. For affine Lie algebras, these are the operators that implement the subtraction of positive roots.

In brief, a bosonic character reflects the construction of an irreducible module starting from a highest-weight Verma module (by which we mean a module generated freely by the application of the lowering operators). Its hallmark are thus not only the $(q)_{\infty}^{-1}$ factors but also the alternating signs in the character expression. 
By contrast, 'fermionic-type' characters or more precisely, fermionic-sum representations have a quite different structure. Unsurprisingly, the prototype is the character of a free-fermion Fock space whose natural product form has the following sum decomposition:

$$
\chi^{\mathrm{ff}}=q^{-1 / 48} \prod_{n=0}^{\infty}\left(1+q^{n+1 / 2}\right)=\sum_{n=0} \frac{q^{n^{2} / 2}}{(q)_{n}}
$$

The rhs is the generating function for partitions in distinct parts - which is appropriate for fermions - and this sum representation is obtained by a simple variant of the Euler identity (cf. eq. (2.2.6) of corollary 2.2 of $[6]$ ):

$$
\prod_{n=0}^{\infty}\left(1+t q^{n}\right)=\sum_{n=0}^{\infty} \frac{t^{n} q^{n(n-1) / 2}}{(q)_{n}}
$$

with $t=q^{1 / 2}$. The sum describes the filling of the space of states in terms of particles each term of the sum representing the contribution of a different particle-number sector -, subject to a restriction rule, here the Pauli exclusion principle. This interpretation of the filling of the space of states in terms of quasi-particles with restriction rules is the characteristic feature of fermionic-sum representations. It generically manifests itself by the presence of $(q)_{n}$ factors in the denominator. But more significantly, it is expressed in the form of a positive $q$ series (i.e., there are no alternating signs). The bosonic counterpart of this fermionic sum is simply the combination of the Rocha-Caridi characters $\chi_{11}+\chi_{21}$.

As another simple example, consider the free-boson theory, whose character is $q^{-1 / 24}(q)_{\infty}^{-1}$, and its representation in terms of two complex fermions. Each complex fermion is described by the partition $q^{-1 / 48} \prod_{n=0}^{\infty}\left(1+q^{n+1 / 2}\right)$. However, in order to keep track of the charge, we introduce an auxiliary label $z$ and represents the two characters as $q^{-1 / 48} \prod_{n=1}^{\infty}\left(1+z q^{n-1 / 2}\right)$ and $q^{-1 / 48} \prod_{n=0}^{\infty}\left(1+z^{-1} q^{n+1 / 2}\right)$ instead. Our free boson theory is recovered from the zero-charge sector of the product of these characters. Using again the Euler identity (1.4), we recover, from this projected product, another well-known identity of Euler (cf. eq. (2.2.5) of corollary 2.2 of [6]):

$$
\frac{1}{(q)_{\infty}}=\sum_{n=0}^{\infty} \frac{q^{n^{2}}}{(q)_{n}^{2}}
$$

The rhs is the fermionic-sum representation of a free-boson character (up to the $q^{-1 / 24}$ factor). For this example, the bosonic counterpart, namely the lhs, is rather simple in that 
there are no singular-vector subtractions. In other words, the bosonic-sum representation reduces to a single term. But the 'bosonic identification mark' $(q)_{\infty}^{-1}$ is present.

Let us consider still another example, namely the Virasoro characters of the Yang-Lee model $\left(p^{\prime}, p\right)=(2,5)$, which reads

$$
\chi_{1,1+a}=q^{(11-12 a) / 60} \sum_{m=0}^{\infty} \frac{q^{m(m+a)}}{(q)_{m}}=q^{(11-12 a) / 60} \prod_{\substack{n=1 \\ n \neq 0,(2-a) \bmod 5}}^{\infty} \frac{1}{\left(1-q^{n}\right)}
$$

with $a=0,1$. The above equalities are nothing but the famous Rogers-Ramanujan-Schur identities (cf. [6], eqs (7.1.6), (7.1.7)). 1 As indicated, this fermionic-sum representation has a natural product version. This result has a natural generalization to the whole $\left(p^{\prime}, p\right)=(2,2 k+1)$ series. 2

\subsection{The origins of fermionic-sum characters in conformal field theory}

The discovery, over the last decade, of a rich class of fermionic-sum representations in conformal field theory has various origins.

1 The Lie algebraic derivation of this relation is given in Ex. 14.8 of [7] and appears to be due to Kac [8]. The product form of these Virasoro characters was discovered independently in [9].

2 Are there analogous bosonic vs fermionic forms for the character expressions in the case of finite algebras? Although a bit far-fetched, the following $s u(2)$ Lie-algebra example indicates that this is indeed so, at least if 'fermionic representation' is understood in the broader sense of 'quasi-particle representation'. For an irreducible representation with finite Dynkin label $\ell$ (i.e., $\ell=2 j$ ), which has dimension $\ell+1$, the Weyl character formula yields

$$
\chi_{(\ell)}=\frac{q^{-\ell}-q^{\ell+2}}{1-q^{2}}=\frac{q^{-\ell}\left(1-q^{2 \ell+2}\right)}{\left(q^{2}\right)_{1}}
$$

This is the bosonic form of the character, that captures the subtraction of one singular vector. On the other hand, the Schwinger's oscillator model of angular momentum algebra, that is, the representation of the algebra in terms of two independent harmonic oscillators (see e.g., [10]), yields the quasi-particle representation:

$$
\chi_{(\ell)}=q^{-\ell} \sum_{\substack{m=0 \\ m-\ell=0 \bmod 2}}^{\ell} q^{2 m}=q^{-\ell} \sum_{\substack{m=0 \\ m-\ell=0 \bmod 2}}^{\ell} \frac{\left(q^{2}\right)^{m}}{\left(q^{2}\right)_{0}}
$$

This is obtained by projecting the product of the charged harmonic oscillator characters $(1-$ $z q)^{-1}\left(1-z^{-1} q\right)^{-1}$ onto the $z^{2 \ell}$ sector. In this case however, the quasi-particles have no fermionic attribute. 


\section{A-Representation theory of Lie algebras}

The first fermionic formulae for conformal characters appeared in mathematics, as the characters of the $Z$ Lepowsky-Wilson algebra [11], which turns out to be equivalent to the parafermionic algebra of Zamolodchikov-Fateev [12]. The resulting characters have been obtained in [13]. By construction, these characters are associated to those of the primary fields of the coset model $\widehat{s u}(2)_{k} / \widehat{u}(1)$, for which bosonic-type formulae already existed - these are the string functions of [2]. That provided the first nontrivial example of bosonic-fermionic character identities. Both sides (bosonic vs fermionic) have direct $\widehat{s u}(N)$ extensions and these are related to generalized parafermionic models [14].

The Lepowsky-Primc formulae, in a slightly modified form, pop up in a second and quite unexpected conformal-field-theoretical context, namely as the characters of $(2,2 k+1)$ minimal models $[8,15]$. For $k=2$, this is related to the result $(1.6)$.

\section{B-Dilogarithm identities}

A different development is rooted in the dilogarithm identities, originating from the TBA analysis of integrable perturbations of conformal field theory [16], relating the central charge and the conformal dimensions. Within the scope of conformal field theory, these identities can be obtained by comparing the asymptotic behavior of the usual bosonic character expressions with their fermionic-sum representations [20], which were known for the $(2,2 k+1)$ models. It has then been suggested that such identities (which are not limited to the $(2,2 k+1)$ series) could somehow be lifted to the whole characters [21], producing characters written naturally as fermionic sums, as in the rhs of the AndrewsGordon identity $[22,23]$

$$
\prod_{n \neq 0, \pm i \bmod 2 k+1} \frac{1}{1-q^{n}}=\sum_{m_{1}, \cdots, m_{k-1}} \frac{q^{N_{1}^{2}+\cdots N_{k-1}^{2}+N_{i}+\cdots+N_{k-1}}}{(q)_{m_{1}} \cdots(q)_{m_{k-1}}}
$$

This program has effectively been realized in [24]. Building on an earlier observation [25] that the complete spectrum of generalized $\widehat{g} / \widehat{u}(1)^{r}$ parafermionic conformal theories steams from the dilogarithm function, the authors have conjectured a natural lift of the

3 Such identities also arise from the low temperature limit of the free energy of integrable lattice systems (RSOS-type spin chains) [17] and in the leading behavior of the lattice transfer matrix [18 ]. For a beautiful review of the various facets of the dilogarithm identities, see [19]. 
asymptotic parafermionic characters in the form of fermion-sum representations that include [13] as a special case.

\section{C- The scaling limit of spin chains}

At the same period, fermionic-sum representations emerge from a completely different perspective. In $[26,27]$, the spectrum of the gapless three-state Potts model has been computed in the conformal limit by solving the Bethe equations. That leads to a description of the hamiltonian eigenspectrum in terms of quasi-particles obeying a fermionic exclusion principle in momentum space, supplemented by further restrictions constraining the momentum eigenvalues. These spectrum computations lead to characters expressed directly as fermionic sums. In the (anti) ferromagnetic sector, the scaling limit of this model is the $\left(\mathbb{Z}_{4}\right) \mathbb{Z}_{3}$ parafermionic theory and the built characters agree with the results of [13]. A key observation at this stage appeared to be the rewriting of the Lepowsky-Primc result in terms of the inverse Cartan matrix for $\widehat{s u}(k)$, which, in turn, suggested a large number of natural generalizations [3, 28, 29, 30], all having an underlying quasi-particle interpretation.

The proof of most these identities has been supplied in the following years. An important step in that direction was the observation [4] that the Virasoro characters have a natural 'finitized version' in terms of path spaces - or corner-transfer-matrix sums - in the RSOS model [31]. That allowed for a proof of the simplest cases; this line of attack has subsequently been developed vigorously, starting with the paper [32].

Few years later, the fermionic-type exclusion principle underlying the quasi-particle filling of the space of states was shown in [33] to be precisely the Haldane's generalization of the Pauli exclusion principle [34].

\section{D- Spinons bases and generalization}

Quasi-particle descriptions of conformal field theories also originate from still another and quite different source. This one is rooted in the discovery of the remarkable connection between the $s u(N)$ Haldane-Shastry model [35] (an integrable spin chain with long-range interaction) and $\widehat{s u}(N)_{1}$ WZW model, the former appearing as a sort of discretization of the latter [36]. This relation yields naturally a spinon description of the WZW basis of states, which has been lifted to fermionic-sum representations for the characters [37, 38].

As an outgrowth of the spinon descriptions of WZW models, a general approach for quasi-particle reformulation of conformal field theories has been presented in [39] (and much 
exemplified in e.g., in [40]). It revolves around the construction of truncated fermionic-type characters.

\subsection{Bosonic vs fermionic parafermionic characters}

Let us return to the parafermionic case. Recently, we have obtained new expressions for the $\mathbb{Z}_{k}$ parafermionic characters [41]. These expressions are different from those obtained by the coset construction, the string functions, themselves identical to the parafermionic characters obtained by the BRST projection of a two-boson representation $[42,43]$. These new character formulae are rooted in the description of the parafermionic states in terms of a standard basis (cf. section 2). The highest-weight modules constructed in the framework of this basis contain an infinite number of singular vectors. The explicit expression of all these singular vectors has been obtained and by their appropriate subtraction and addition, we have constructed bosonic-type parafermionic characters [41]. The resulting formula is reported in appendix $\mathrm{A}$ in a somewhat improved form.

Given these new expressions, it is natural to try to establish directly their equivalence to the ones in [13]. However, a frontal attack is difficult, even in the two extreme cases $k=2$ (the Ising model) and the $k=\infty$ (the two complex bosons limit - cf. appendix B). That suggested us to look instead for a reformulation of our standard basis in the form of a new fermionic-type basis that would be expressed in terms of the modes of a single type of parafermionic field complemented with specific restriction rules. The aim of this paper is to present that new basis, derived from a purely parafermionic perspective.

We complete this introduction with a brief presentation of the paper's content. The next section is intended to fix the notation and present the standard basis of [41]. The new basis is displayed in section 3. It is formulated in terms of simple restriction rules. These rules are first motivated by inductive considerations and heuristic arguments relying on an exclusion principle for $\mathbb{Z}_{k}$ quasi-particles. The selection rules are then shown to be rooted in the $\mathbb{Z}_{k}$ invariance. Technical details establishing the genuine character of the proposed basis are reported in an appendix. The basis is then illustrated for various

4 Actually, they do not have a purely bosonic appearance in that they have no $(q)_{\infty}^{-1}$ factors and they contain fermionic ingredients, namely $(q)_{n}$ terms in the denominators - cf. appendix A. However, their alternating-sign structure qualifies them as genuine bosonic sums. Note that their direct analytic equivalence with the string functions has not been established yet. 
simple modules in section 4. In section 5, this basis is lifted to the general expression of the $\mathbb{Z}_{k}$ characters, written in terms of a positive sum over restricted partitions. This result is then reexpressed in product form using Andrews identity. We obtain complete agreement with the Lepowsky-Primc formula [13].

\section{The standard basis of the $\mathbb{Z}_{k}$ parafermionic modules}

The $\mathbb{Z}_{k}$ parafermionic conformal algebra $[12,44]$ is generated by the parafermionic fields $\psi_{r}=\psi_{k-r}^{\dagger}, r=0,1, \cdots, k-1$, of conformal dimension $h_{\psi_{r}}=r(k-r) / k$. Since the higher-order parafermions and their conjugate can be obtained from composition of the fundamental ones, $\psi_{1}$ and $\psi_{1}^{\dagger}$, it suffices to write the commutation relations involving the modes of these fundamental parafermions. The mode decomposition of $\psi_{1}$ and $\psi_{1}^{\dagger}$ acting on a generic field of charge $\phi_{q}$ reads:

$$
\begin{aligned}
& \psi_{1}(z) \phi_{q}(0)=\sum_{m=-\infty}^{\infty} z^{-q / k-m-1} A_{(1+q) / k+m} \phi_{q}(0) \\
& \psi_{1}^{\dagger}(z) \phi_{q}(0)=\sum_{m=-\infty}^{\infty} z^{q / k-m-1} A_{(1-q) / k+m}^{\dagger} \phi_{q}(0)
\end{aligned}
$$

the fractional power of $z$ being fixed by the mutual locality [12]. Note that the dimension of a parafermionic mode is the negative of its index.

Since the fractional part of the modes is fixed unambiguously by the charge of the field or state on which it acts, it can be omitted. To indicate that the fractional part has been deleted (which implies that the conformal dimension of the mode is no longer given by minus its index), we use calligraphic symbols, 5 i.e.,

$$
\mathcal{A}_{n}\left|\phi_{q}\right\rangle \equiv A_{n+(1+q) / k}\left|\phi_{q}\right\rangle, \quad \mathcal{A}_{n}^{\dagger}\left|\phi_{q}\right\rangle \equiv A_{n+(1-q) / k}^{\dagger}\left|\phi_{q}\right\rangle
$$

Given that the parafermions $\psi_{1}, \psi_{1}^{\dagger}$ have respective charge 2 and -2 , the modes $\mathcal{A}_{n}$ and $\mathcal{A}_{n}^{\dagger}$ also have charge 2 and -2 . When reinserting the fractional part of the modes in a given string of operators, these charges must be taken into account. For instance, we have

$$
\left(\mathcal{A}_{-1}\right)^{3}\left|\phi_{q}\right\rangle \equiv A_{-1+(5+q) / k} A_{-1+(3+q) / k} A_{-1+(1+q) / k}\left|\phi_{q}\right\rangle
$$

5 This is the notation used in [41] except that there $\mathcal{B}$ was used to denoted $\mathcal{A}^{\dagger}$ and the symbol $N$ played the role of $k$. 
With this simplifying notation, the commutation relation reads then $[12,41]$

$$
\begin{aligned}
& \sum_{l=0}^{\infty} C_{-2 / k-1}^{(l)}\left[\mathcal{A}_{n-l-1} \mathcal{A}_{m+l+1}^{\dagger}+\mathcal{A}_{m-l}^{\dagger} \mathcal{A}_{n+l}\right] \phi_{[q, \bar{q}]}(0) \\
& =\left[\frac{(k+2)}{k} L_{n+m}+\frac{1}{2}\left(n+\frac{q}{k}\right)\left(n-1+\frac{q}{k}\right) \delta_{n+m, 0}\right] \phi_{[q, \bar{q}]}(0)
\end{aligned}
$$

and

$$
\sum_{l=0}^{\infty} C_{2 / k}^{(l)}\left[\mathcal{A}_{n-l} \mathcal{A}_{m+l}-\mathcal{A}_{m-l} \mathcal{A}_{n+l}\right] \phi_{[q, \bar{q}]}(0)=0
$$

together with a similar relation with $\mathcal{A} \rightarrow \mathcal{A}^{\dagger}$ and where $C_{t}^{(l)}=\Gamma(l-t) / l ! \Gamma(-t)$.

In order to describe the standard basis of states, we need to recall the definition of a parafermionic highest-weight state [12]. For a fixed value of $k$, there are $k$ parafermionic primary fields $\left\{\varphi_{\ell} \mid \ell=0, \cdots, k-1\right\} ; \varphi_{\ell}$ has charge $\ell$ and dimension $h_{\ell}=\ell(k-\ell) / 2 k(k+2)$. To each primary field, there corresponds a highest-weight state $\left|\varphi_{\ell}\right\rangle$, with $|0\rangle=\left|\varphi_{0}\right\rangle$. The parafermionic highest-weight conditions are

$$
\mathcal{A}_{n}\left|\varphi_{\ell}\right\rangle=\mathcal{A}_{n+1}^{\dagger}\left|\varphi_{\ell}\right\rangle=0 \quad \text { for } \quad n \geq 0
$$

The set of independent states at level $s$ in the highest-weight module of $\left|\varphi_{\ell}\right\rangle$ of charge $\ell+2 r$ is generated by the following states [41]:

$$
\mathcal{A}_{-n_{1}} \mathcal{A}_{-n_{2}} \ldots \mathcal{A}_{-n_{j}} \mathcal{A}_{-m_{1}}^{\dagger} \mathcal{A}_{-m_{2}}^{\dagger} \ldots \mathcal{A}_{-m_{p}}^{\dagger}\left|\varphi_{\ell}\right\rangle
$$

with

$$
j-p=r, \quad n_{i} \geq n_{i+1} \geq 1, \quad m_{i} \geq m_{i+1} \geq 0, \quad \sum_{i=1}^{j} n_{i}+\sum_{i=1}^{p} m_{i}=s
$$

In the standard basis, we can thus order separately the $\mathcal{A}$ strings and the $\mathcal{A}^{\dagger}$ strings but there is no mixed ordering.

The highest-weight module of $\left|\varphi_{\ell}\right\rangle$ can be decomposed into a direct sum of $k$ modules of fixed charge defined modulo $2 k$. In the highest-weight module of charge $\ell+2 r$ (or relative charge $2 r$ ), the highest-weight state is

$$
\left|\varphi_{\ell}^{(r)}\right\rangle=\left(\mathcal{A}_{-1}\right)^{r}\left|\varphi_{\ell}\right\rangle \quad(0 \leq r \leq k-\ell)
$$


Similarly, if $r<0$, the charged highest-weight state reads

$$
\left|\varphi_{\ell}^{(-r)}\right\rangle=\left(\mathcal{A}_{0}^{\dagger}\right)^{r}\left|\varphi_{\ell}\right\rangle \quad 0 \leq r \leq \ell
$$

The upper bounds for the values of $r$ are induced by the presence of the 'primary' singular vectors:

$$
\left|\chi_{\ell}\right\rangle=\mathcal{A}_{-1}^{k-\ell+1}\left|\varphi_{\ell}\right\rangle, \quad\left|\chi_{\ell}^{\prime}\right\rangle=\left(\mathcal{A}_{0}^{\dagger}\right)^{\ell+1}\left|\varphi_{\ell}\right\rangle
$$

Not all the states $\left|\varphi_{\ell}^{( \pm r)}\right\rangle$ are independent however; there is the following field identification [45]:

$$
\varphi_{\ell}^{(-\ell)} \sim \varphi_{\ell}^{(k-\ell)} \sim \varphi_{k-\ell} \equiv \varphi_{\ell}^{\dagger}
$$

This implies that either $\left\{\varphi_{\ell}^{(r)}\right\}$ or $\left\{\varphi_{\ell}^{(-r)}\right\}$ can be chosen as a complete set of basic parafermionic fields (which correspond to the highest weights in a fixed charge module). In other words, a complete set of charged highest-weight states is generated from the parafermionic primary fields by applying either strings of $\mathcal{A}$ or $\mathcal{A}^{\dagger}$ operators; at this level, the use of both types of modes is thus superfluous. As it will be shown in the next section, this can also be made true for all the descendants.

\section{A new parafermionic basis and the underlying exclusion principle}

\subsection{The quasi-particle basis}

We now look for a fermionic-type basis for the parafermionic theories. By a fermionictype basis, we mean a basis that is free of singular vectors, akin to the free-fermion basis, and in which the states in a module are filled subject to simple restrictions. More precisely, singular vectors should be eliminated by a selection rule rather than being subtracted.

Since there is an infinite number of singular vectors in the standard basis, it would appear highly improbable that they can all be taken into account by means of simple restrictions. This suggest that a fermionic-type basis will not be naturally formulated in terms of both the $\mathcal{A}$ and $\mathcal{A}^{\dagger}$ modes but rather in terms of a single type, say $\mathcal{A}$. This is certainly possible given that $\psi_{1}^{\dagger}=\psi_{k-1} \sim\left(\psi_{1}\right)^{k-1}$. In a single mode-type basis, the constraint of a fixed value for the difference on the number of $\mathcal{A}$ and $\mathcal{A}^{\dagger}$ operators in a module of fixed charge is replaced by the conservation of the number of $\mathcal{A}$ operators modulo $k$. 
In a fermionic-type basis expressed in terms of the $\mathcal{A}$ modes only, states in the highestweight module of $\left|\varphi_{\ell}\right\rangle$ of relative charge $2 r$ will naturally be written as ordered strings of the form

$$
\mathcal{A}_{-n_{1}} \mathcal{A}_{-n_{2}} \cdots \mathcal{A}_{-n_{m}}\left|\varphi_{\ell}\right\rangle \quad \text { with } \quad n_{i} \geq n_{i+1} \geq 1
$$

with $m=r \bmod k$. In addition, we have to take into account the presence of the singular vector $\left|\chi_{\ell}\right\rangle$ - cf. (2.11). This gives an upper bound on the possible number of $\mathcal{A}_{-1}$ factors at the right end of the string. Since the $n_{i}$ 's are ordered and all greater or equal to 1 , this requirement is fully captured by the simple condition:

$$
n_{m-k+\ell} \geq 2
$$

That ensures that the $k-\ell+1$-th operator counted from the right is not $\mathcal{A}_{-1}$. The set (3.1) with the restriction (3.2) still generates too much states, meaning that the specification of the basis requires further selection rules.

The missing selection rules are the following. All ordered sequences of $\mathcal{A}$ operators that contain any one of the following $k$-string

$$
\left(\mathcal{A}_{-(n+1)}\right)^{k-i}\left(\mathcal{A}_{-n}\right)^{i} \quad(i=0, \cdots, k-1)
$$

must be forbidden. Together with the condition (3.2), these restrictions imposed on an ordered sequence of operators provide a basis. Note that when $\ell=0,1$, the condition (3.2) is superfluous, being taken into account by the above restrictions.

In view of writing down the characters, that is, of counting the number of states per level, it is convenient to reformulate the above restriction rules algebraically as follows:

$$
n_{j}-n_{j+k-1} \geq 2
$$

It is somewhat surprising that this simple condition captures precisely the whole set of restrictions encoded in (3.3).

The next two sections serve to establish the naturalness of the restrictions rules (3.3), using first an inductive approach and then a sort of generalized exclusion principle. The restriction rules are then traced back to the mere $\mathbb{Z}_{k}$ invariance. That precisely the states (3.3) can be eliminated as a result of the $\mathbb{Z}_{k}$ invariance is proved in appendix $\mathrm{C}$. 


\subsection{Inductive derivation of the restriction rules}

To justify the naturalness of the restriction rules (3.3), we first 'derive' them following an inductive line. The $k=2$ parafermionic theory is a free-fermion model. A natural starting point is thus to first retranslate the fermionic Fock basis into the $\mathcal{A}$-mode language, and then look for the generalization to $k>2$.

Since the parafermionic field is a free fermion when $k=2$, the standard fermionic modes $b_{-\nu}$ are nothing but the $A_{-\nu}$ modes (keeping track of their fractional part - cf. [41] appendix C), e.g.,

$$
b_{-n+1 / 2}|0\rangle=A_{-n+1 / 2}|0\rangle=\mathcal{A}_{-n}|0\rangle
$$

The fermionic Fock basis is

$$
b_{-\nu_{1}} b_{-\nu_{2}} \cdots b_{-\nu_{m}}\left|\varphi_{\ell}\right\rangle \quad \text { with } \quad \nu_{i}>\nu_{i+1}
$$

where $\ell=0,1$. With $\nu_{i}=n_{i}-f_{i}$, where $f_{i}$ stands for the fractional part, it is directly translated into

$$
A_{-n_{1}+(1+\ell+2 p-2) / 2} \cdots A_{-n_{m}+(1+\ell) / 2}\left|\varphi_{\ell}\right\rangle \quad \text { with } \quad n_{i}-f_{i}>n_{i+1}-f_{i+1}
$$

After being reexpressed in terms of the integer-mode operators $\mathcal{A}$, in the form (3.1), we see that the ordering will be preserved if $n_{i}>n_{i+1}$. The fermionic nature of the $\mathcal{A}$ modes is itself taken into account by the condition $n_{i}>n_{i+1}+1$. In other words, in any ordered sequence of $\mathcal{A}$ modes, we exclude the following two subsequences:

$$
\mathcal{A}_{-(n+1)} \mathcal{A}_{-n} \quad \text { and } \quad \mathcal{A}_{-n} \mathcal{A}_{-n}
$$

The $k=2$ version of our new basis will then be spanned by all states of the form

$$
\mathcal{A}_{-n_{1}} \mathcal{A}_{-n_{2}} \cdots \mathcal{A}_{-n_{m}}\left|\varphi_{\ell}\right\rangle \quad \text { with } \quad n_{i} \geq n_{i+1}+2
$$

Note that the condition (3.2) is taken into account by the above difference 2 condition.

Consider now the $k=3$ model. The crucial step is finding the proper specification of the sequences that should be forbidden. For $k=3$, these will be sequences of three $\mathcal{A}$ modes. The most natural generalization of the exclusion (3.8) amounts to forbid the following 3-strings

$$
\mathcal{A}_{-n} \mathcal{A}_{-n} \mathcal{A}_{-n}, \quad \mathcal{A}_{-(n+1)} \mathcal{A}_{-n} \mathcal{A}_{-n}, \quad \mathcal{A}_{-(n+1)} \mathcal{A}_{-(n+1)} \mathcal{A}_{-n}
$$

These are obtained by inserting $\mathcal{A}_{-n}$ or $\mathcal{A}_{-(n+1)}$ at either end of (3.8). Similarly, the appropriate $k=4$ forbidden 4 -states are:

$$
\begin{array}{ll}
\mathcal{A}_{-n} \mathcal{A}_{-n} \mathcal{A}_{-n} \mathcal{A}_{-n}, & \mathcal{A}_{-(n+1)} \mathcal{A}_{-n} \mathcal{A}_{-n} \mathcal{A}_{-n}, \\
\mathcal{A}_{-(n+1)} \mathcal{A}_{-(n+1)} \mathcal{A}_{-n} \mathcal{A}_{-n}, & \mathcal{A}_{-(n+1)} \mathcal{A}_{-(n+1)} \mathcal{A}_{-(n+1)} \mathcal{A}_{-n}
\end{array}
$$

The generalization (3.3) is then immediate. 


\subsection{A quasi-particle reinterpretation of the effective parafermionic exclusion principle}

Forbidding the states (3.3) is equivalent to implementing an effective $\mathbb{Z}_{k}$ parafermionic exclusion principle. This interpretation is made more precise in the present subsection.

Let us come back to the Pauli exclusion principle: two fermions cannot be put in the same state and this translates into the statement $b_{-\nu} b_{-\nu}=0$. The natural generalization is obvious: we cannot put $k \mathbb{Z}_{k}$-parafermions in the same state. This however lacks precision: what is the right meaning of 'the same state'? The state characterization should refer to the type of excitation. But this can be labeled by the complete mode index of the creation operator (i.e., including its fractional part), - which is minus the conformal dimension, referred to here as the energy -, or by its integral part. But 'the same state' characterization cannot pertain to the energy since the energy label always increases along a string (e.g., the fractional part increases steadily by steps of $2 / k$ from right to left). Hence, it has to refer to the integral part of the mode. But the exclusion rule itself must also be related to the energy of the states. In that regard, the energy of a state of $n$ identical excitations will refer to the average energy.

We thus formulate the following 'principle': in the $\mathbb{Z}_{k}$ model, we cannot add $p \mathcal{A}_{-n}$

excitations to a state that already contains $(k-p)$ quasi-particles of type $\mathcal{A}_{-m}$, with $n \geq m$, unless the average energy of the $n$ modes is strictly greater than that of the $m$ modes. We now show that this forbids of the $k$-strings listed in (3.3). Consider the string

$$
\left(\mathcal{A}_{-n}\right)^{p}\left(\mathcal{A}_{-m}\right)^{k-p}=A_{-\nu_{1}} \cdots A_{-\nu_{p}} A_{-\nu_{p+1}} \cdots A_{-\nu_{k}} \quad\left(\nu_{i}=n_{i}-f_{i}\right)
$$

acting on a state of ahcrge $q$. The average energy of the $\mathcal{A}_{-n}$ and the $\mathcal{A}_{-n}$ modes is respectively

$$
\mathcal{E}_{n}=n-2+\frac{p-q}{k}, \quad \mathcal{E}_{m}=m-1+\frac{p-q}{k}
$$

so that $\mathcal{E}_{n}>\mathcal{E}_{m}$ implies $n>m+1$, and this is equivalent to the exclusion of the complete set of $k$-strings listed in (3.3).

This of course is not a proof of the selection rule (3.3) but simply a reformulation in a quasi-particle language. 


\subsection{On the 'effective' nature of the exclusion principle and its $\mathbb{Z}_{k}$ origin}

For $k>2$, the $\mathbb{Z}_{k}$ 'effective exclusion principle' (3.3) cannot be a genuine exclusion principle as in the $k=2$ case. For this, it would be necessary that at least one $k$-string (3.3) be exactly zero in analogy with the $k=2$ case, where one of the two sequences (3.8) vanishes in view of the fermionic nature of the $\mathcal{A}$ modes. It is simple to check, through examples, that the action of these $k$-strings is generically not equal to zero. For instance, for $k=3$, we can check that $\mathcal{A}_{-2} \mathcal{A}_{-2} \mathcal{A}_{-1}|0\rangle \neq 0$ and $\mathcal{A}_{-3} \mathcal{A}_{-2} \mathcal{A}_{-2}|0\rangle \neq 0$. The meaning of the restriction rule is confined to a restriction on the counting of states: all states containing $k$-strings of the type (3.3) can be reexpressed in terms of other states which are free of them.

Now, what is the origin of this restriction? It is bound to be rooted in the mere $\mathbb{Z}_{k}$ invariance, namely the relation $\left(\psi_{1}\right)^{k} \sim \mathbb{I}$. Up to this point this condition has not been taken into account properly, having been invoked only to argue that the charge of the descendent states is defined modulo $2 k$. But this condition means that at every level, one needs to take out one state in each k-string. This is precisely what the restriction rule (3.3) does for us. That these conditions imposed on an ordered sequences indeed provide a basis, is demonstrated in appendix $\mathrm{C}$.

\section{Illustrative examples}

In this section, we display the states at the first few levels in some simple vacuum modules for $k=2$ and 3. But before, we clarify the relation between the conformal dimension of a string of $\mathcal{A}$ operators and the sum of its integer modes.

\subsection{The fractional dimension of a p-string}

Consider the module of $\left|\varphi_{\ell}\right\rangle$ of relative charge $2 r$. The various states all contain $m=$ $k j+r \mathcal{A}$ operators acting on $\left|\varphi_{\ell}\right\rangle$. The level $s$ appropriate to a given sequence is obtained by summing the mode indices (with reversed sign) and subtracting the contribution of the sum of the omitted fractional parts. Explicitly, the $A$-version of the string reads

$$
A_{-n_{1}+(1+2(m-1)+\ell) / k} \cdots A_{-n_{m}+(1+\ell) / k}\left|\varphi_{\ell}\right\rangle
$$


The fractional parts $f_{i}$ add up to

$$
F=\sum_{i=1}^{k j+r} f_{i}=k j^{2}+2 j r+j \ell+\frac{r^{2}}{k}+\frac{\ell r}{k}
$$

The dimension of the string is then $\sum n_{i}-F$. In the $r=0$ charge sector, this dimension is integer. The character is constructed by summing the number of states at each level $s$ and by multiplying the result by $q^{h_{\ell}-c / 24}$, with $c=2(k-1) /(k+2)$. In a charged sector, the dimension of the string is not integral. But if we redefine $s$ as

$$
s^{\prime}=s+\frac{r^{2}}{k}+\frac{\ell r}{k}-r
$$

$s^{\prime}$ is integer and the shift amounts to modify the prefactor from $q^{h_{\ell}-c / 24}$ to $q^{h_{\ell}^{(r)}-c / 24}$ where $h_{\ell}^{(r)}$ stands for the dimension of the basic field $\varphi_{\ell}^{(r)}$, associated to the highest-weight state in the charge $2 r$ sector:

$$
h_{\ell}^{(r)}=h_{\ell}+\frac{r(k-\ell-r)}{k}
$$

Summarizing, the states at level $s^{\prime}$ in the module of $\left|\varphi_{\ell}\right\rangle$ of relative charge $2 r$, are generated by the decompositions of the integer $n$ related to $s^{\prime}$ by

$$
n=\sum_{i=1}^{j k+r} n_{i}=s^{\prime}+k j^{2}+j \ell+(2 j+1) r
$$

into $k j+r$ parts subject to the selection rules (3.2) and (3.3). In the following, the states will be represented by the corresponding reversed partitions, that is,

$$
\mathcal{A}_{-n_{1}} \mathcal{A}_{-n_{2}} \cdots \mathcal{A}_{-n_{m}}\left|\varphi_{\ell}\right\rangle \quad \rightarrow \quad\left(n_{m}, \cdots n_{1}\right)
$$

\subsection{The $k=2$ case}

Consider first the uncharged 6 vacuum $(\ell=r=0)$ module of the Ising model. All the states are then constructed by the application of an even number of ordered $\mathcal{A}$ modes, forbidding pairs whose mode index do not differ by at most 2 . In other words, we consider

6 The use of the word 'charge' in the context of the Ising model is of course formal since there is no physical notion of charge when $k=2$. 
partitions into an even number of parts that differ by at least 2 . The vacuum itself is represented by the void partition and there is no state at level 1 . The list of states at the next first few levels is

$$
\begin{array}{ll}
s=2: & (13) \\
s=3: & (14) \\
s=4: & (15) \quad(24) \\
s=5: & (16) \quad(25) \\
s=6: & (17) \quad(26) \quad(35) \\
s=7: & (18)(27) \quad(36) \\
s=8: & (19) \quad(28) \quad(37) \quad(46) \quad(1357) \\
s=9: & (1,10)(29) \quad(38) \quad(47) \quad(1358) \\
s=10: & (1,11) \quad(2,10) \quad(39) \quad(48) \quad(57) \quad(1359) \quad(1368)
\end{array}
$$

It is clear that the minimum level at which a string of length $2 j$ occurs is $2 j^{2}$.

The charged sector of the vacuum module $(\ell=0, r=1)$ describes the Virasoro freefermion module. It is obtained from the vacuum by the application of an odd number of $\mathcal{A}$ operators. The first state is $\mathcal{A}_{-1}|0\rangle$, of dimension $1 / 2$. If we redefine the dimension of the other states with respect to this highest state in the charged sector, we have then

$$
\begin{array}{ll}
s^{\prime}=0:(1) & s^{\prime}=4:(5) \quad(135) \\
s^{\prime}=1:(2) & s^{\prime}=5:(6) \quad(136) \\
s^{\prime}=2:(3) & s^{\prime}=6:(7) \quad(137) \quad(246) \\
s^{\prime}=3:(4) & s^{\prime}=7:(8)(138) \quad(147) \quad(146)
\end{array}
$$

The remaining character is that of the spin field, which corresponds here to the case $\ell=1$. Its charged and uncharged modules are equal, the $r=1$ case describing the disorder field. The partitions associated to the first few states with $r=0$ are:

$$
\begin{aligned}
& s=1:(13) \quad s=4:(16)(25) \\
& s=2:(14) \quad s=5:(17)(26) \quad(35) \\
& s=3:(15)(24) \quad s=6: \text { (18) (27) (36) (1357) }
\end{aligned}
$$

Of course the partitions appearing here are identical to those already seen in the vacuum module. The only difference is that the threshold level for the appearance of partitions of lengths $2 j$ is shifted by a value that depends upon $j$. 


\subsection{The $k=3$ case}

For the uncharged vacuum module of the three-states Potts model, the list of the first few states obtained by the application of $3 j$-strings excluding the three 3 -strings (3.10), is

$$
\begin{aligned}
& s=2:(113) \\
& s=3:(114)(123) \\
& s=4:(115)(124)(133) \\
& s=5:(116)(125)(134)(224) \\
& s=6:(117) \quad(126) \quad(135) \quad(144) \quad(225) \quad(234) \quad(113355) \\
& s=7 \text { : (118) (127) (136) (145) (226) (236) (244) (113356) } \\
& s=8 \text { : (119) (128) (137) (146) (155) (227) (236) (245) (335) } \\
& \text { (113357) (113366) (113456) }
\end{aligned}
$$

Up to level 14, the number of states in the 3-, 6- and 9- particle states are given by

$\begin{array}{cccccccccccccccc}s & 0 & 1 & 2 & 3 & 4 & 5 & 6 & 7 & 8 & 9 & 10 & 11 & 12 & 13 & 14 \\ p[3] & & & 1 & 2 & 3 & 4 & 6 & 7 & 9 & 11 & 13 & 15 & 18 & 20 & 23 \\ p[6] & & & & & & & 1 & 1 & 3 & 5 & 9 & 13 & 21 & 28 & 41 \\ p[9] & & & & & & & & & & & & & & & 1\end{array}$

where $p[3 j]$ stands for the number of partitions of the integer $s+3 j^{2}$ into $3 j$ parts that forbid the subpartitions corresponding the 3 -strings (3.10). The 9-particle sector starts then at level $s=14$, corresponding to the partition (113355779). This counting of state can be compared to the sum of Virasoro characters $\chi_{0}^{(3)}=q^{1 / 30}\left(\chi_{11}+\chi_{41}\right)$ of the $\left(p^{\prime}, p\right)=(5,6)$ minimal model.

The two charged sectors $(r=1,2)$ yield the modules of the two parafermionic fields (which are equivalent), and the counting of states reproduces that of the Virasoro field $\phi_{13}$. Similarly, with $\ell=1,2$, the spin-field character $\chi_{23}$ is recovered and the charged sector yields the energy-field module $\chi_{21}+\chi_{31}$.

\section{Fermionic-type parafermionic character formulae}

\subsection{Character formulae in terms of restricted partitions}

The various states in the highest-weight module $\left|\varphi_{\ell}\right\rangle$ of relative charge $2 r$ are those obtained by the application of $r+j k \mathcal{A}$ operators on the highest-weight state, forbidding 
(3.3) and keeping track of the singular vector $\left|\chi_{\ell}\right\rangle$ in (2.11). The counting of the resulting states level by level for a fixed value of the relative charge yields the parafermionic highestweight characters.

Denote by $p_{\Delta_{k, \ell}}^{[m]}(n)$ the number of partitions of $n=n_{1}+\cdots+n_{m}$ into $m=j k+r$ parts subject to the constraints:

$$
\Delta_{k} n_{i} \equiv n_{i}-n_{i+k-1} \geq 2, \quad n_{i} \geq n_{i+1} \geq 1, \quad n_{m-k+\ell} \geq 2
$$

Such partitions will be called $\Delta_{k, \ell}$-partitions.

The $\varphi_{\ell}$-character in the relative charge sector $2 r$ is thus (cf. section 4.1 )

$$
\chi_{\ell, r}=q^{h_{\ell}^{(r)}-c / 24} \sum_{s^{\prime}=0}^{\infty} \sum_{j=0}^{\infty} q^{s^{\prime}} p_{\Delta_{k, \ell}}^{[j k+r]}\left(s^{\prime}+j^{2} k+j \ell+(2 j+1) r\right)
$$

where $h_{\ell}^{(r)}$ is given by (4.4). In particular, for the vacuum in the zero charge sector $(\ell=r=0)$, it takes the simpler form

$$
\chi_{0,0}=q^{-c / 24} \sum_{s=0}^{\infty} \sum_{j=0}^{\infty} q^{s} p_{\Delta_{k, \ell}}^{[j k]}\left(s+j^{2} k\right)
$$

Equation (5.2) is a truly remarkable result in that all the $\mathbb{Z}_{k}$ parafermionic characters, in all possible charge sectors, are written in terms of a single uniform expression. In a fixed charged sector, all the characters are determined by exactly the same restricted partitions, their level contribution being simply shifted differently for different values of $\ell$.

The expression (5.2) reflects the quasi-particle nature of the underlying basis in that it involves no subtraction. However it is not yet written in a fermionic-sum representation. This last step is done in the next subsection.

\subsection{Character formulae in fermionic-sum representation}

In order to obtain the fermionic form of the character (5.3), we need to construct the generating function for the $\Delta_{k, \ell}$-partitions. The counting of such partitions at fixed length can be reformulated into the counting of the number of solutions of simple Diophantine inequalities. The construction of the generating functions for such problems is well-known (see for instance [46]) and it is used in appendix D to build up the $k=2$ product form 
of the character (5.3) and that of the 3-particle sector of the Potts vacuum character. However, this method gets quickly cumbersome.

Fortunately, the solution of this precise general problem has already been obtained by Andrews [22] (see also [6]) and in this section we use directly that result. The number of $\Delta_{k, \ell}$-partitions of $n$ into $m$ parts that contain at most $k-\ell$ parts equal to 1 is given by (cf. Theorem 1 of [47] with $d=0$ and $i=k-\ell+1-$ or eq. (7.38) of [6])

$$
\sum_{n, m=0}^{\infty} p_{\Delta_{k, \ell}}^{[m]}(n) q^{n} z^{m}=\sum_{m_{1}, \cdots, m_{k-1}=0}^{\infty} \frac{q^{N_{1}^{2}+\cdots+N_{k-1}^{2}+L_{k-\ell+1}} z^{N}}{(q)_{n_{1}} \cdots(q)_{n_{k-1}}}
$$

where

$$
\begin{array}{ll}
N=N_{1}+\cdots+N_{k-1}, & N_{i}=m_{i}+\cdots+m_{k-1} \\
L_{j}=N_{j}+\cdots N_{k-1}, & L_{k}=L_{k+1}=0
\end{array}
$$

Let us apply this to the general case of a charged character of $\varphi_{\ell}$. Recall that the partitions we are interested in are related to the level $s^{\prime}$ (itself related to $s$ by (4.3)) via $n=s^{\prime}+j^{2} k+(2 j+1) r$. In order to count $j k+r$-string partitions, we set $z^{m}=0$ if $m$ is not equal to $r \bmod k$. For $m=j k+r$, we will fix $z^{m}$ in order to adjust the power of $q$ in our sum over $n$ to that of the level $s$, namely, to transform

$$
\sum_{n} p_{\Delta_{k, \ell}}^{[j k+r]}\left(s^{\prime}+j^{2} k+j \ell+(2 j+1) r\right) q^{n} z^{m} \rightarrow \sum_{s} p_{\Delta_{k, \ell}}^{[j k+r]}\left(s^{\prime}+j^{2} k+j \ell+(2 j+1) r\right) q^{s^{\prime}}
$$

For this we need to set

$$
z^{m}=\left\{\begin{array}{cc}
q^{-(m-r)(m+r+\ell) / k-r} & \text { when } m=j k+r \\
0 & \text { otherwise }
\end{array}\right.
$$

The identity (5.4) becomes then

$$
\begin{aligned}
& \sum_{s^{\prime}, j=0}^{\infty} p_{\Delta_{k, \ell}}^{[j k+r]}\left(s^{\prime}+j^{2} k+j \ell+(2 j+1) r\right) q^{s^{\prime}} \\
& \quad=\sum_{\substack{m_{1}, \cdots, m_{k-1}=0 \\
N=r \bmod k}}^{\infty} \frac{q^{N_{1}^{2}+\cdots+N_{k-1}^{2}-(N-r)(N+r+\ell) / k-r+L_{k-\ell+1}}}{(q)_{m_{1}} \cdots(q)_{m_{k-1}}}
\end{aligned}
$$

Note that on the rhs, the contribution to the $j k$-particle sector is selected by the condition $N=\sum i m_{i}=j k$. Multiplied by $q^{h_{\ell}^{(r)}-c / 24}$, this yields the general form of the $\mathbb{Z}_{k}$ charged characters in a fermionic-sum representation. With this factor, this is precisely the Lepowsky-Primc formula (theorem 9.4 of [13]). 


\section{Conclusion}

We have presented a new basis for the space of states in $\mathbb{Z}_{k}$ parafermionic conformal field theories. It yields a 'faithful' description of the states in irreducible modules, faithful in the sense that it does not require the explicit subtraction of an infinite number of singular-vectors 0 The basis is formulated in terms of a single type of modes, namely, the modes $\mathcal{A}$ of the parafermionic field $\psi_{1}$. States in a highest-weight module are thus of the form $\mathcal{A}_{-n_{1}} \cdots \mathcal{A}_{-n_{m}}\left|\varphi_{\ell}\right\rangle$. The various constraints on the mode-label $n_{i}$ implement in a very natural way:

(1) - an ordering: $n_{i} \geq n_{i+1}$;

(2) - the highest-weight nature of $\left|\varphi_{\ell}\right\rangle: n_{i} \geq 1$;

(3) - the subtraction of one 'primary' singular vector, the one that can be formulated solely in terms of the $\mathcal{A}$ modes - cf. $\left|\chi_{\ell}\right\rangle$ in (2.11) - and this is taken into account by the 'boundary condition': $n_{p-k+\ell} \geq 2$;

(4) - the $\mathbb{Z}_{k}$ invariance, which implies that $\left(\psi_{1}\right)^{k} \sim \mathbb{I}$, which is taken care of by the forbidding of the $k$-strings (3.3), or equivalently, the $\Delta_{k}$ condition: $n_{i}-n_{i+k-1} \geq 2$. As explained in section 3 , this condition can be formulated in terms of an effective exclusion principle.

The counting of partitions of $n=n_{1}+\cdots+n_{m}$ subject to these conditions (called $\Delta_{k, \ell}$-partitions) leads directly to the $\mathbb{Z}_{k}$ characters written in a manifestly positive series expansion in $q$ (cf. eq. (5.2)). Using Andrews' identity (5.4), the generating function of these restricted partitions can in turn be written as sums of products. The resulting character formula (5.8) is precisely the one obtained by Lepowsky-Primc [13].

Since a character expression is the transcription of a basis, our new fermionic-type basis is bound to be equivalent to that of [13]. Actually, our last step (namely, section 5.2 ) is identical to the final step in the derivation of the Lepowsky-Primc formula: we count the same type of objects since at this point, our basis and theirs turn out to be equivalent. The precise equivalence is obtained when their $z_{\alpha}(-n)$ operators are replaced

7 The existence of such a basis was not expected in the initial stage of our study of parafermionic theories - cf. the discussion following eq (1.1) of [41]. 
by our $\mathcal{A}_{-n}$ ones (cf. their theorem 6.8 and the constraints (6.13)). 8 However, their approach, which is Lie-algebraic, is completely different from ours. Their original aim was to obtain representations of standard modules in $\widehat{s u}(2)_{k}$ by constructing $z_{\alpha}$ operators in the so-called homogeneous picture, meaning that they are forced to commute with those of the homogeneous Heisenberg algebra, itself simply a $\widehat{u}(1)$ algebra. Given that the $\mathbb{Z}_{k}$ parafermionic theory has a natural description in terms of the coset $\widehat{s u}(2)_{k} / \widehat{u}(1)$, it is clear, a posteriori, that the $z_{\alpha}$ operators have to be directly related to the parafermionic fields. The present results give the precise correspondence mentioned above.

Our basis is also equivalent to the $\mathbb{Z}_{k}$ quasi-particle basis displayed in [40], but presented there in a more complicated-looking form (cf. their eqs (4.3)-(4.5)), which is further valid only for the vacuum, and given without proof - apart from the plain fact that it yields the right character.9

Although at the end, we reproduce the Lepowsky-Primc basis in a disguised form, we stress that there is a great interest for a purely parafermionic derivation of this fermionictype basis. Firstly, the parafermionic point of view is clearly more physical, having a natural quasi-particle interpretation. In this spirit, we have seen that the $\Delta_{k}$ condition comes from our effective $\mathbb{Z}_{k}$ exclusion principle, which in turn reflects the built-in $\mathbb{Z}_{k}$ invariance, through $\left(\psi_{1}\right)^{k} \sim \mathbb{I}$. Secondly, there exist generalized parafermionic theories that do not have a simple coset description of the type $\widehat{g} / \widehat{u}(1)^{r}$ - to which the LepowskyPrimc construction should be limited. This is certainly the case for the $\mathbb{Z}_{k}^{(\beta)}$ models in which the dimension of the parafermionic field $\psi_{n}$ reads $\beta n(k-n) / k$, with $\beta$ integer - cf. appendix A of [12]). The search for a quasi-particle basis in those cases requires purely parafermionic techniques such as the ones considered here. 10

On the other hand, we stress that the quasi-particle basis displayed here is not the unique quasi-particle basis underlying $\mathbb{Z}_{k}$ parafermionic conformal theories. Indeed, as

8 We stress in that regard that the transition from fractional to integer modes for writing of strings of parafermionic modes clarified the way for the formulation of the standard basis in [41] and also the fermionic-type basis presented here; and it is with this notation that the connection with [13] becomes crystal clear.

9 Their second vacuum basis (their eq. (4.10)) appears to be a mere rearrangement of the first one and it is not proved either.

10 In that vein, it should be noticed that in a parafermionic approach, it is somewhat natural to first unravel the bosonic form of the character given that it contains some information (namely one 'primary' singular vector) needed in the formulation of the quasi-particle basis. 
stressed in [3], there are two different integrable models whose scaling limit is the $\mathbb{Z}_{k}$ parafermionic theories: the $s u(k+2)$ RSOS model at the boundary of regimes I/II and the $\mathbb{Z}_{k}$ spin chains [48] and these have different quasi-particles description. Actually, a second fermionic sum representation has been found in $[29,30]$. It would be interesting to unravel the structure of the underlying basis. In that vein, it would be of interest to connect each fermionic sum to a particular integrable perturbation by an energy operator [49].

We conclude with some general comments. The first one pertains to the AndrewsGordon-Rogers-Ramanujan-Schur identities themselves. The classical Rogers-RamanujanSchur relations (1.6) - which correspond to (1.7) for $k=2$ - have the following combinatorial interpretation (see e.g., [6] corollaries 7.6 and 7.7): the number of partitions of $n$ into $m$ numbers such that $n_{i}-n_{i+1} \geq 2$, with the 'boundary condition' $n_{m} \geq 1+a$, is equal to the number of partitions of $n$ into parts not congruent to $0, \pm(2-a) \bmod 5$. The most immediate generalization that one could think of is to replace the 2 in the difference condition by a larger integer. Surprisingly, that faces various no-go theorems (cf. the introduction of [23]). The breakthrough for generalizations came when Gordon considered the Glaisher's $k$-periodicity condition, i.e., a condition on the difference $n_{i}-n_{i+k-1}$, together with the lower bound 2. The analysis presented here allows us to state that one side of the combinatorics underlying these classical Rogers-Ramanujan-Schur identities has a very natural fermionic field-theoretical interpretation. In that vein, the corresponding side of the Andrews-Gordon generalization similarly has a quite natural parafermionic interpretation 11 in that it constrains $k$ adjacent parts along a sort of $\mathbb{Z}_{k}$ exclusion principle.12

The pervasive nature of the $\Delta_{k}$ condition is quite impressive. In conformal field theory, this ubiquity is itself signaled in the many occurrences of fermionic-type character formulae that have appeared recently and whose core is precisely this $\Delta_{k}$ condition. And this condition reflects a $\mathbb{Z}_{k}$ invariance. It might be a hint that parafermions could provide

11 The 'boundary' part of this statement should be slightly qualified, however. The RogersRamanujan-Schur 'boundary condition' is not exactly the one which is appropriate to the Ising model and this generalizes the higher $k$ cases. Both (combinatorial and parafermionic) conditions would be identical if the singular vector $\left|\chi_{\ell}\right\rangle$ was given by $\mathcal{A}_{-1}^{k-\ell}\left|\varphi_{\ell}\right\rangle$ instead of $\mathcal{A}_{-1}^{k-\ell+1}\left|\varphi_{\ell}\right\rangle$.

12 We found afterwards that similar comments are presented in [50], where this observation appears as a motivation for studying $Z$-algebras. See also the introduction of [51]. In the last chapter of this book, the authors also give the correspondence between the $Z$-algebras and the $\mathbb{Z}_{k}$ parafermionic theory. 
the core description of a priori quite unrelated models. In particular, it is rather remarkable to see that the characters of the $(2,2 k+1)$ minimal models are also of the Lepowsky-Primc form [15], which strongly suggests an underlying parafermionic construction.

\section{Appendix A. The bosonic-type $\mathbb{Z}_{k}$ character formula}

The character of the highest-weight module $\varphi_{\ell}$ with relative charge $2 t \geq 013$ is given by $[41]$

$$
\chi_{\ell, t}=q^{h_{\ell}^{(t)}-c / 24-t} \sum_{s=0} q^{s} g_{\ell, t}(s)
$$

where $h_{\ell}^{(t)}$ is given by (4.4) and $g_{\ell, t}(s)$ corresponds to the number of states at level $s$ having charge $2 t$ and it is given by

$$
g_{\ell, t}(s)=\sum_{p=0}^{\infty} g_{\ell, t, p}(s)
$$

with

$$
g_{\ell, t, p}(s)=G_{0, t} \delta_{p, 0}-G_{r_{p-1}, s_{p}+t}-G_{r_{p}^{\prime}, s_{p-1}^{\prime}+t}+G_{r_{p}, s_{p}+t}+G_{r_{p}^{\prime}, s_{p}^{\prime}+t}
$$

where

$$
G_{a, b}(s) \equiv \sum_{j=0}^{s} \sum_{s_{1}=j}^{s} p^{(j-a)}\left(s_{1}-j\right) p^{(j-b)}\left(s-s_{1}\right)
$$

with $a, b$ two non-negative integers. The indices of $G$ in (A.3) are defined as follows:

$$
\begin{array}{ll}
r_{p}=(p+1)[(p+1)(k+2)+\ell+1], & \left.r_{p}^{\prime}=(p+1)\right)[(p+1)(k+2)-\ell-1] \\
s_{p}=(p+1)[(p(k+2)+\ell+1], & s_{p}^{\prime}=(p+1)[(p+2)(k+2)-\ell-1]
\end{array}
$$

In (A.4), $p^{(j-a)}(n)$ stands for the partition of $n$ into at most $j$ parts 14 and

$$
p^{(0)}(n)=\delta_{n, 0}, \quad p^{(j \geq 0)}(0)=1, \quad p^{(j<0)}(n)=0
$$

which implies in particular that $G_{a, b}(s)=0$ if $s<\max (a, b)$. Simple illustrations of this formula can be found in section 6 of [41].

13 Recall that in view of the field identifications, it suffices to consider the non-negative charge sector only.

14 For instance, $p^{(3)}(6)=7$ since 6 can be decomposed in 7 different ways in sums of at most three integers: $6=5+1=4+2=3+3=4+1+1=3+2+1=3+1+1=2+2+2$. 
These character formulae can be written somewhat more compactly in terms of the generating function for the factors $\sum q^{s} G_{a, b}(s)$. Notice that

$$
\sum_{s=0}^{\infty} q^{s} G_{a, b}(s)=\sum_{s=0}^{\infty} \sum_{j=0}^{s} \sum_{s_{1}=j}^{s} q^{s} p^{(j-a)}\left(s_{1}-j\right) p^{(j-b)}\left(s-s_{1}\right)
$$

With the conditions $\left(\right.$ A.6) and $p^{(j)}(n<0)=0$, we can start the sum over $s_{1}$ from 0 , extend that of $j$ to $\infty$ and reexpress that over $s=s_{1}+s_{2}$ as one over $s_{2}$, with the result:

$$
\sum_{s=0}^{\infty} q^{s} G_{a, b}(s)=\sum_{j=0}^{\infty}\left(\sum_{s_{1}=0}^{\infty} q^{s_{1}} p^{(j-a)}\left(s_{1}-j\right)\right)\left(\sum_{s_{2}=0}^{\infty} q^{s_{2}} p^{(j-b)}\left(s_{2}\right)\right)=\sum_{j=0}^{\infty} \frac{q^{j}}{(q)_{j-a}(q)_{j-b}}
$$

The occurrence of the $(q)_{n}$ factors makes these characters sorts of hybrid between bosonic and fermionic forms.

\section{Appendix B. Bosonic vs fermionic characters of the $\mathbb{Z}_{\infty}$ model}

In the $k \rightarrow \infty$ limit, the parafermionic theory reduces to a theory of two independent complex bosons [52]. In that limit, the bosonic form of the parafermionic character becomes rather simple in that there is a single contributing singular vector, namely $\left(\mathcal{A}_{0}^{\dagger}\right)^{\ell+1}\left|\varphi_{\ell}\right\rangle$.

Let us consider for simplicity the vacuum module of zero relative charge, in which case the single contributing singular vector arises at level 1 . The $r=0$ vacuum character reduces then to (with $c=2$ ):

$$
\chi_{0,0}=q^{-1 / 12} \sum_{s=0}^{\infty} q^{s}\left[G_{0,0}(s)-G_{0,1}(s)\right]
$$

This will be shown to be equivalent to the character of the vacuum module in a theory of two independent complex bosons, with modes $a_{n}$ and $\bar{a}_{n}$, when evaluated in the zerocharge sector. The highest-weight conditions being $a_{n}|0\rangle=\bar{a}_{n}|0\rangle=0$ for $n \geq 0$, the different states at level $s$ in the uncharged vacuum module are of the form

$$
a_{-n_{1}} \cdots a_{-n_{j}} \bar{a}_{-m_{1}} \cdots \bar{a}_{-m_{j}}|0\rangle \quad \text { with } \quad n_{i} \geq n_{i+1}, \quad m_{i} \geq m_{i+1}
$$

and

$$
\sum_{i=1}^{j} n_{i}=s_{1} \quad \sum_{i=1}^{j} m_{i}=s-s_{1}
$$


The corresponding character is simply

$$
\chi_{0}^{\mathrm{cb}}=q^{-1 / 12} \sum_{s=0}^{\infty} q^{s} \sum_{j=0}^{\infty} \sum_{s_{1}=j}^{\infty} p^{[j]}\left(s_{1}\right) p^{[j]}\left(s-s_{1}\right)
$$

where, as usual, $p^{[j]}(n)$ stands for the number of partitions of $n$ into exactly $j$ parts.

Let us now demonstrate that (B.1) reduces to (B.4). For this we simply note that

$$
\begin{aligned}
G_{0,0}(s)-G_{0,1}(s) & =\sum_{j=0}^{\infty} \sum_{s_{1}=j}^{\infty} p^{(j)}\left(s_{1}-j\right)\left[p^{(j)}\left(s-s_{1}\right)-p^{(j-1)}\left(s-s_{1}\right)\right] \\
& =\sum_{j=0}^{\infty} \sum_{s_{1}=j}^{\infty} p^{(j)}\left(s_{1}-j\right) p^{[j]}\left(s-s_{1}\right) \\
& =\sum_{j=0}^{\infty} \sum_{s_{1}=j}^{\infty} p^{[j]}\left(s_{1}\right) p^{[j]}\left(s-s_{1}\right)
\end{aligned}
$$

Since $p^{(j)}(n)$ is the number of partitions of $n$ into at most $j$ parts, it is clear that $p^{(j)}(n)-$ $p^{(j-1)}(n)=p^{[j]}(n)$. In the last line, we used the relation $p^{(j)}(n-j)=p^{[j]}(n)$. 1. The number of states at level $s$ is thus the same as in the vacuum module of the two-complexboson theory.

The character (B.4) can easily be put in a fermionic-type form. The generating function of a free-boson character is simply $q^{-1 / 24}(q)_{\infty}^{-1}$. To take the charge into account, we introduce a parameter $z$ into the Euler function:

$$
(z ; q)_{\infty} \equiv \prod_{n=1}^{\infty}\left(1-z q^{n}\right)
$$

States in the product Fock space of two complex bosons are thus generated by the product $q^{-1 / 12}\left[(z ; q)_{\infty}\left(z^{-1} ; q\right)_{\infty}\right]^{-1}$. To get the complete set of states of zero charge, we project this expression onto the $z^{0}$ sector. Using the Euler relation (cf. theorem 2.1 of [6] with $a=0$ and $t=z q)$ :

$$
\frac{1}{(z ; q)_{\infty}}=\sum_{j=0}^{\infty} \frac{(z q)^{j}}{(q)_{j}}
$$

15 The respective generating functions of these partitions are

$$
\sum_{j=0}^{\infty} p^{(j)}(n) q^{n}=\frac{1}{(q)_{j}} \quad \sum_{j=0}^{\infty} p^{[j]}(n) q^{n}=\frac{q^{j}}{(q)_{j}}
$$

from which the above identity follows directly. 
it ready follows that

$$
\left.\frac{1}{(z ; q)_{\infty}\left(z^{-1} ; q\right)_{\infty}}\right|_{z^{0}}=\sum_{j=0}^{\infty} \frac{(q)^{2 j}}{(q)_{j}^{2}}
$$

so that

$$
\chi_{0}^{\mathrm{cb}}=q^{-1 / 12} \sum_{j=0}^{\infty} \frac{(q)^{2 j}}{(q)_{j}^{2}}
$$

Of course, the above sum is nothing but (cf. (A.8)):

$$
\sum_{s=0}^{\infty} q^{s}\left[G_{0,0}(s)-G_{0,1}(s)\right]=\left(\frac{q^{j}}{(q)_{j}(q)_{j}}-\frac{q^{j}}{(q)_{j}(q)_{j-1}}\right)
$$

The expression (B.9) is rather different from (5.8) evaluated in the $k \rightarrow \infty$ limit and we have not made the direct correspondence. Note however that there are many ways that even the Euler function $(q)_{\infty}^{-1}$ for instance can be transformed into a multiple $q$-series (cf. theorem 3 of [47]):

$$
\frac{1}{(q)_{\infty}}=\sum_{m_{1}, \cdots, m_{k-1}} \frac{q^{N_{1}^{2}+\cdots N_{k-1}^{2}}}{(q)_{m_{1}} \cdots(q)_{m_{k-2}}(q)_{m_{k-1}}^{2}}
$$

In this identity, the number $k$ is totally arbitrary (and with $k=2$, this reduces to (1.5)).

It is also interesting to recall the form of the vacuum string function in that limit $[2$, 42, 43]:

$$
b_{0}^{(\infty)}=q^{-1 / 12} \frac{1}{(q)_{\infty}^{2}}\left[1+2 \sum_{p=1}^{\infty}(-1)^{p} q^{p(p+1) / 2}\right]
$$

The equivalence of this expression with (B.4) provides a nice identity.

\section{Appendix C. From the $\mathbb{Z}_{k}$ invariance to the restriction rules}

In this appendix, we prove that, as a result of the model's $\mathbb{Z}_{k}$ invariance, the $k$ distinct $k$-strings (3.3) can be forbidden. Before plunging into the details, we outline the argument.

At first, we rephrase the condition $\left(\psi_{1}\right)^{k} \sim \mathbb{I}$ in terms of modes of the $k$-th composite parafermionic field $\psi_{k}$ and argue that this implies one linear relation at each level. This condition is then reexpressed in an ordered basis formulated solely in terms of the $\mathcal{A}$ modes. We show that this naturally amounts to forbid the product of $k$ strings $\mathcal{A}_{-n_{1}} \cdots \mathcal{A}_{-n_{k}}$ that 
satisfy $n_{k}+1 \geq n_{1}$. This inequality selects precisely the $k$-strings (3.3) and this set indeed generates one forbidden state at each level in product of $k \mathcal{A}$ descendant operators.

In a second step, the compatibility requirements of these selection rules imposed within strings of length $m>k$ are studied. At this level, we must ensure that there are no induced contradictions and that exactly the right number of constraints is still generated. Arguing from that perspective shows that the selection rules (3.3) are singled out in that they forbid sequences of states with indices 'as equal as possible'.

We thus start by displaying the commutation relations between the modes of the parafermions $\psi_{r}$ and $\psi_{s}$, whose OPE reads

$$
\psi_{r}(z) \psi_{s}(w) \sim \frac{c_{r, s}}{(z-w)^{2 r s / k}} \psi_{r+s}(w) \quad(r+s \leq k)
$$

with

$$
c_{r, s}^{2}=\frac{(r+s) !(k-r) !(k-s) !}{r ! s !(k-r-s) ! k !}
$$

With $\psi_{r}$ expanded in modes as

$$
\psi_{r}(z) \phi_{q}(0)=\sum_{m=-\infty}^{\infty} z^{-r q / k-m-r} A_{r(r+q) / k+m}^{(r)} \phi_{q}(0)
$$

where $\phi_{q}$ is an arbitrary field of charge $q$, the mode commutation relation between $A_{u}^{(r)}$ and $A_{v}^{(s)}$ reads

$$
\sum_{l=0}^{\infty} C_{2 r s / k-1}^{(l)}\left[\mathcal{A}_{n-l-r}^{(r)} \mathcal{A}_{m+l-s+1}^{(s)}+\mathcal{A}_{m-l-s}^{(s)} \mathcal{A}_{n+l-r+1}^{(r)}\right]=c_{r, s} \mathcal{A}_{n+m-r-s+1}^{(r+s)}
$$

In this last expression, the field $\phi_{q}$ and the fractional parts of the modes have been omitted; $C_{t}^{(l)}$ is defined after $(2.5)$.

Since $\left(\psi_{1}\right)^{k} \sim \mathbb{I}$, the modes $\mathcal{A}_{p}^{(k)}$ are in fact simply delta functions. Being interested in relations between states, we thus write $\mathcal{A}_{p}^{(k)} \sim 0$. That yields one condition for every value of $p$. By setting $r=k-1, s=1, m=-n_{k}$ and $n=-n_{1}-\cdots-n_{k-1}+k-1$ in (C.4), we find

$$
\sum_{l=0}^{\infty} C_{1-2 / k}^{(l)} \mathcal{A}_{-n_{1}-\cdots-n_{k-1}-l}^{(k-1)} \mathcal{A}_{-n_{k}+l} \sim-\sum_{l=0}^{\infty} C_{1-2 / k}^{(l)} \mathcal{A}_{-n_{k}-l-1} \mathcal{A}_{-n_{1}-\cdots-n_{k-1}+l+1}^{(k-1)}
$$


We now use (C.4) recursively to eliminate the modes of the composite parafermions $\mathcal{A}^{(k-i)}$ starting from $i=1$ up to $i=k-2$. Note that the precise manner in which $\mathcal{A}_{n}^{(k)}$ is first decomposed into two parts $\mathcal{A}_{n-m}^{\left(k_{1}\right)} \mathcal{A}_{m}^{\left(k_{2}\right)}$ with $k_{1}+k_{2}=k$ (here $k_{1}, k_{2}$ being chosen to be 1 and $k-1$ ), and then in which the various $\mathcal{A}_{n}^{(k-i)}$ are further split, does not influence the final result in view of associativity. In other words, the different ways of separating $\mathcal{A}_{n}^{(k)}$ do not provide different relations.

The splitting of the modes in the initial decomposition (C.5) has been chosen in such a way that the lhs can produce the term $\mathcal{A}_{-n_{1}} \mathcal{A}_{-n_{2}} \cdots \mathcal{A}_{-n_{k}}$ (using (C.4) recursively) for which we assume that the indices $n_{i}$ 's are ordered: $n_{1} \geq n_{2} \geq \cdots \geq n_{k}$. Let us isolate this term on the lhs. It can thus be expressed as a linear combination of the form $\sum c_{1 \cdots k} \mathcal{A}_{-n_{1}^{\prime}} \mathcal{A}_{-n_{2}^{\prime}} \cdots \mathcal{A}_{-n_{k}^{\prime}}$, with $\sum n_{i}=\sum n_{i}^{\prime}$. These terms are supposed to have been reordered after the decomposition. Now all these ordered terms are necessarily independent of $\mathcal{A}_{-n_{1}} \mathcal{A}_{-n_{2}} \cdots \mathcal{A}_{-n_{k}}$ if it happens that the negative of their first mode index is strictly greater than that of $\mathcal{A}_{-n_{1}} \mathcal{A}_{-n_{2}} \cdots \mathcal{A}_{-n_{k}}$, i.e., than $n_{1}^{\prime}>n_{1}$. Now if $n_{1}^{\prime}=n_{1}$, it is clear that $\mathcal{A}_{-n_{1}} \mathcal{A}_{-n_{2}^{\prime}} \cdots \mathcal{A}_{-n_{k}^{\prime}}$ is not necessarily independent of our isolated term and for this, we obviously need to compare the index with smallest $i$ such that $n_{i}^{\prime} \neq n_{i}$.

Enforcing that minus the first index of every term on the rhs be greater or equal to $n_{1}$, leads to a sequence of constraints which we now derive. For this, we need to write down all possible terms which occur in the decomposition of the two sides of (C.5). If the leftmost operator is already of the $\mathcal{A}$ type, no further analysis is required. This is the case for the rhs of (C.5). On the lhs, let us redefine $l \rightarrow l_{1}$. Then, by using (C.4) once more, we get (do not caring about relative coefficients)

$$
\begin{aligned}
\mathcal{A}_{-n_{1}-\cdots-n_{k-1}-l_{1}}^{(k-1)} & \sim \mathcal{A}_{-n_{1}-\cdots-n_{k-2}-l_{1}-l_{2}}^{(k-2)} \mathcal{A}_{-n_{k-1}+l_{2}} \\
& +\mathcal{A}_{-n_{k-1}-l_{2}-1} \mathcal{A}_{-n_{1}-\cdots-n_{k-2}-l_{1}+l_{2}+1}^{(k-2)}
\end{aligned}
$$

The second term on the rhs has the desired form (an $\mathcal{A}$ factor at the left). We then need to transform the first one. It is clear that this first term, upon complete decomposition with similar index splitting, will generate the term $\mathcal{A}_{-n_{1}} \mathcal{A}_{-n_{2}} \cdots \mathcal{A}_{-n_{k}}$. Moreover, at each step of the process, there will be a term produced whose leftmost contribution will be $\mathcal{A}_{-n_{k-i}-l_{i+1}-1}$. Recall that it is the mode index of all these leftmost terms that need to be compared with $n_{1}$. This leads to the following list of constraints, in their most stringent version (i.e., with $l_{i+1}=0$ ):

$$
n_{i}+1 \geq n_{1}
$$


Since the $n_{i}$ 's are supposed to be ordered, all these constraints are consequences of a single one, namely

$$
n_{k}+1 \geq n_{1}
$$

Quite remarkably, if all these constraints are enforced and that $n_{1}^{\prime}=n_{1}$, then it follows that $n_{i}^{\prime}=n_{i}$ for all other $i . \mathcal{A}_{-n_{1}} \mathcal{A}_{-n_{2}} \cdots \mathcal{A}_{-n_{k}}$ itself is thus recovered and it is transferred to the lhs. This shows that when all the constraints are satisfied on the first index of the prime terms, $\mathcal{A}_{-n_{1}} \mathcal{A}_{-n_{2}} \cdots \mathcal{A}_{-n_{k}}$ can be eliminated.

The condition (C.8) eliminates exactly one state at every level. Actually, it selects precisely the $k$ terms listed in (3.3).

We now investigate the compatibility requirement associated to the elimination of the $k$-strings (3.3) when they are immersed within strings of length $m>k$. Let us forget for a moment the special structure of the $k$ strings that have been selected by the above analysis and focus on the plain fact that there is one constraint at each level that results from the $\mathbb{Z}_{k}$ invariance. Suppose that we select one such constraint at each level in some well-prescribed way. The point we want to stress here is that there is no guarantee that these eliminated states, when considered within longer strings, will yield the correct number of constraints.

Take the $k=3$ uncharged vacuum module. At each level we have to eliminate one state. We could select the one with largest value of $n_{1}$. At $s=3$, that would amount to eliminate $\mathcal{A}_{-4} \mathcal{A}_{-1} \mathcal{A}_{-1}$. Now consider the $r=1$ vacuum module. At $s^{\prime}=6$ we get the following states:

$$
\begin{aligned}
& s^{\prime}=6: \quad \mathcal{A}_{-5} \mathcal{A}_{-1} \mathcal{A}_{-1} \mathcal{A}_{-1} \quad \mathcal{A}_{-4} \mathcal{A}_{-2} \mathcal{A}_{-1} \mathcal{A}_{-1} \quad \mathcal{A}_{-3} \mathcal{A}_{-3} \mathcal{A}_{-1} \mathcal{A}_{-1} \\
& \mathcal{A}_{-3} \mathcal{A}_{-2} \mathcal{A}_{-2} \mathcal{A}_{-1} \quad \mathcal{A}_{-2} \mathcal{A}_{-2} \mathcal{A}_{-2} \mathcal{A}_{-2}
\end{aligned}
$$

In this context, the elimination of the 3 -string $\mathcal{A}_{-4} \mathcal{A}_{-1} \mathcal{A}_{-1}$ is problematic since we do not encounter the state $\mathcal{A}_{-2} \mathcal{A}_{-4} \mathcal{A}_{-1} \mathcal{A}_{-1}$. Hence, not enough constraints are generated.

The $k$ distinct $k$-strings that are eliminated in the quasi-particle basis have the noteworthy structure of being precisely those products of $k$ operators whose mode indices are 'as equal as possible'. This has the important implication that within any ordered sequence of operators, any one of the $k$ sequences (3.3) cannot appear separated, that is, no reordering is required for bringing together a sequence of a $k$-string of the type (3.3). In our example, it means that we eliminate $\mathcal{A}_{-2} \mathcal{A}_{-2} \mathcal{A}_{-2}$ at level 3 and this state is found either in the form $(\mathcal{A A A}) \mathcal{A}$ or $\mathcal{A}(\mathcal{A} \mathcal{A} \mathcal{A})$ in any ordered sequence of 4 -strings. 
Is it possible that too many constraints be generated? Suppose that we eliminate $\mathcal{A}_{-2} \mathcal{A}_{-2} \mathcal{A}_{-1}$ at $s=2$ and $\mathcal{A}_{-3} \mathcal{A}_{-3} \mathcal{A}_{-1}$ at $s=4$. In the $s^{\prime}=6$ list (C.9), we would then eliminate both $\mathcal{A}_{-3} \mathcal{A}_{-3} \mathcal{A}_{-1} \mathcal{A}_{-1}$ and $\mathcal{A}_{-3} \mathcal{A}_{-2} \mathcal{A}_{-2} \mathcal{A}_{-1}$. But a few lines computation shows that $\mathcal{A}_{-3} \mathcal{A}_{-2} \mathcal{A}_{-2} \mathcal{A}_{-1} \sim \mathcal{A}_{-3} \mathcal{A}_{-3} \mathcal{A}_{-1} \mathcal{A}_{-1}$. That is, reshuffling the indices of an excluded state generates another excluded state. Here it would thus suffice to eliminate only one of $\mathcal{A}_{-2} \mathcal{A}_{-2} \mathcal{A}_{-1}$ or $\mathcal{A}_{-3} \mathcal{A}_{-3} \mathcal{A}_{-1}$ to eliminate the proper number of terms at level $s^{\prime}=6$. This is a circumstance in which too many states are eliminated as a result of a non-compatible choice of excluded 3-strings.

Now, that the states which are eliminated are those with indices 'as equal as possible' guarantees the compatibility of the elimination process itself. The transformation of such a state $\mathcal{A}_{-n_{1}} \mathcal{A}_{-n_{2}} \cdots \mathcal{A}_{-n_{m}}$ into other ordered states implies a redistribution of the integers $\left(n_{1}, n_{2}, \cdots, n_{m}\right)$ into $\left(n_{1}^{\prime}, n_{2}^{\prime}, \cdots, n_{m}^{\prime}\right)$. In this way, $n_{m}$ cannot be increased and the new states are obtained by modifying the labels successively from right to left. Starting the process with a state including a substring in the list (3.3), it is guaranteed that no state containing either this substring or another substring in this list can be encountered later in the process. In other words, the linear decomposition is triangular and thus manifestly coherent.

Let us finally comment on the linear dependence of the states (3.1), disregarding for the moment the restrictions (3.4). That the modes can be ordered in that way - up to possible additional constrains - is proved in [41]. Now, these ordered states are easily proved to be independent. Consider first 2-strings and see if there exists some constants $a_{n_{1}, n_{2}}$ (not all zero) such that $\sum a_{n_{1}, n_{2}} \mathcal{A}_{-n_{1}} \mathcal{A}_{-n_{2}}=0$ for $n_{1}+n_{1}=N$ and $n_{1} \geq n_{2} \geq 1$. Using the commutation relations, the term with largest value of $n_{1}$ can be written as a linear combination of the other ordered states plus a number of non-ordered states (with computable non-zero finite coefficients). Hence, there are no $a_{n_{1}, n_{2}}$ 's such that the above sum vanishes. For longer ordered strings, the argument is similar. Consider a subgroup of ordered $p$-strings at a given level and suppose that there is a unique term with largest value of $n_{1}$. Commuting the first two $\mathcal{A}$ 's necessarily generates one non-ordered state whose second index is $-n_{1}$ (and the argument with 2 -strings shows that it cannot vanish). Further commutations can only displace this $-n_{1}$ index along the string. Therefore there are terms that cannot be reordered and this implies the linear independence of such a set of ordered states. Now if in our subgroup of ordered strings, the state with largest value of $n_{1}$ is not unique, we repeat the argument with the index $n_{j}$ that orders the various 
strings with $n_{i}=n_{i}^{\prime}, i=1, \cdots, j-1$ (e.g., $n_{j}>n_{j}^{\prime}$ ). This completes the proof of the linear independence of the states (3.1). Imposing the restriction (3.4) cannot spoil this linear independence: it only amounts to forbid one state per level.

\section{Appendix D. Simple examples of generating functions for $\Delta_{k, \ell}$-partitions from the MacMahon method}

In this appendix, we construct the simplest generating functions for the $\Delta_{k, \ell^{-}}$ partitions, mainly in order to get a more intuitive handle on this counting problem. A $\Delta_{k, \ell}$-partition of $n$ of length $p$ is characterized by the set of inequalities (5.1). Finding the generating function for all partitions $p_{\Delta_{k, \ell}}^{[m]}(n)$ with $j$ and $k$ fixed is equivalent to count the number of solutions to this system of inequalities. This problem, in turn, can be tackled by the MacMahon algorithm for constructing the generating function of the solutions of a system of linear Diophantine inequalities. For a detailed presentation, see section VIII of vol. 2 in [46].

To illustrate the method, let us first consider the simplest possible situation where $k=2, \ell=0, j=1$ and $r=0$ (and write $\Delta_{k, 0}=\Delta_{k}$ ). These correspond to the partitions of $n=s+2$ in two numbers $n_{1}+n_{2}$ subject to the conditions

$$
n_{1} \geq n_{2}+2, \quad n_{2} \geq 1
$$

Consider then the function

$$
G_{1}^{(2)}=\frac{\lambda_{1}^{-2} \lambda_{2}^{-1} q^{-2}}{\left(1-\lambda_{1} q\right)\left(1-\lambda_{2} \lambda_{1}^{-1} q\right)}=\sum_{n_{1}, n_{2} \geq 0} \lambda_{1}^{n_{1}-n_{2}-2} \lambda_{2}^{n_{2}-1} q^{n_{1}+n_{2}-2}
$$

Manifestly, the projection of this function onto positive powers of $\lambda_{1}$ and $\lambda_{2}$ yields the desired generating function once we set $\lambda_{1}=\lambda_{2}=1$. Let us introduce the MacMahon projection symbol $\Omega$, defined by

$$
\stackrel{\lambda}{\Omega} \sum_{n=-\infty}^{\infty} c_{n} \lambda^{n}=\left.\sum_{n \geq 0} c_{n} \lambda^{n}\right|_{\lambda=1}=\sum_{n \geq 0} c_{n}
$$

For these projection manipulations, we use systematically identities of the following type:

$$
\begin{aligned}
\frac{1}{(1-\lambda q)\left(1-\lambda^{-1} q\right)} & =\frac{1}{\left(1-q^{2}\right)}\left(\frac{1}{1-\lambda q}+\frac{\lambda^{-1} q}{1-\lambda^{-1} q}\right) \\
& =\frac{1}{\left(1-q^{2}\right)}\left(\frac{\lambda q}{1-\lambda q}+\frac{1}{1-\lambda^{-1} q}\right)
\end{aligned}
$$


a proper choice of which being often an important simplifying factor (see for instance [53]).

Let us consider

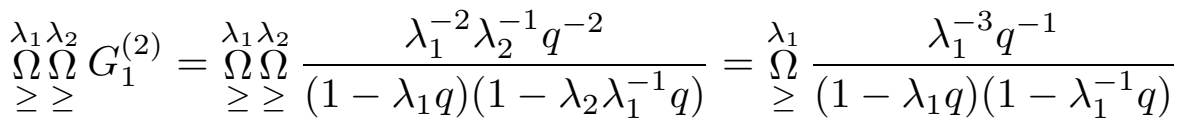

$$
\begin{aligned}
& =\stackrel{\Omega}{\Omega}_{\geq}^{\lambda_{1}} \lambda_{1}^{-3} q^{-1} \frac{1}{\left(1-q^{2}\right)}\left(\frac{\lambda_{1} q}{\left(1-\lambda_{1} q\right)}+\frac{1}{\left(1-\lambda_{1}^{-1} q\right)}\right) \\
& =\stackrel{\lambda}{\Omega}_{\geq}^{\lambda_{1}} \frac{\lambda_{1}^{-2}}{\left(1-q^{2}\right)\left(1-\lambda_{1} q\right)}=\frac{q^{2}}{(1-q)\left(1-q^{2}\right)}=\frac{q^{2}}{(q)_{2}}
\end{aligned}
$$

(recall that after the $\lambda_{i}$ projection, we set $\lambda_{i}=1$ ). In the second line, we can drop the second term because it only contains strictly negative powers of $\lambda_{1}$. This is thus the generating function for the $\Delta_{2}$-partitions into 2 parts.

To count the number of $\Delta_{2}$-partitions with $2 j$ parts, we have to project the function

$$
G_{j}^{(2)}=\lambda^{2 j} q^{-2 j^{2}} \prod_{i=1}^{2 j} \frac{\lambda_{i}^{-2}}{\left(1-\lambda_{i} \lambda_{i-1}^{-1} q\right)}
$$

(with $\lambda_{0}=1$ ) onto non-negative powers of the various $\lambda_{i}$ 's. If we ignore the powers of $\lambda_{i}$ in the numerator, it amounts to neglect the $\Delta_{2}$ constraints as well as the strict positivity of the $n_{i}$ 's. The above projection would then lead directly to the generating function of the partitions of $n$ into at most $2 j$ parts, which reads $(q)_{2 j}^{-1}$. The factors of $\lambda_{i}$ in the numerators are then easily taken into account: they simply generate the term $q^{2 j^{2}}$ in the numerator, leading to the result:

$$
\sum_{s} p_{\Delta_{2}}^{[j k k}\left(s+2 j^{2}\right) q^{s}=\frac{q^{2 j^{2}}}{(q)_{2 j}}
$$

By summing over $j$, we recover the Lepowsky-Primc expression (5.8) for the Ising uncharged vacuum character, or equivalently, (1.3) with $n \rightarrow 2 j$.

Consider now a somewhat less trivial example, the counting of the partitions $p_{\Delta_{3}}^{[3]}(s+3)$. This is associated to the following system of Diophantine inequalities:

$$
n_{1} \geq n_{2} \quad n_{2} \geq n_{3} \geq 1 \quad n_{1} \geq n_{3}+2
$$

for $s+3=n_{1}+n_{2}+n_{3}$. The generating function for the solutions of this system is given by the projection of

$$
G_{1}^{(3)}=\frac{\mu^{-2} \lambda_{3}^{-1} q^{-3}}{\left(1-\mu \lambda_{1} q\right)\left(1-\lambda_{2} \lambda_{1}^{-1} q\right)\left(1-\lambda_{3} \lambda_{2}^{-1} \mu^{-1} q\right)}
$$


onto positive powers of the $\lambda_{i}$ 's and of $\mu$. A simple analysis along the above lines leads to

$$
\sum_{n} p_{\Delta_{3}}^{[3]}(s+3) q^{s}=\frac{q^{2}\left(1+q-q^{3}\right)}{(q)_{3}}
$$

This can be compared to the restriction of (5.8) for $k=3$ in the 3-particle sector, which corresponds to the terms $\left(m_{1}, m_{2}\right)$ with $m_{1}+2 m_{2}=3$, namely $(3,0)$ and $(1,1)$; these add up to

$$
\frac{q^{2}}{(q)_{1}(q)_{1}}+\frac{q^{6}}{(q)_{3}}=\frac{q^{2}\left(1+q-q^{3}\right)}{(q)_{3}}
$$

The coefficients of the $q$-expansion of this expression up to level 14 reproduce those listed in the second row of (4.11).

The higher-particle sectors can be treated by the same technique but the computations become quickly very complicated.

\section{Acknowledgment}

We thank professor J. Lepowsky for clarifying comments concerning his works and for pointing to us references $[50,51]$. This work was supported by NSERC.

\section{REFERENCES}

1. A. Rocha-Caridi, in Vertex Operators in Mathematics and Physics, Eds J. Lepowsky et al, Publ. Math. Sciences Res. Inst. 3, Springer-Verlag, (1985) 451.

2. V. Kac and D. Peterson, Adv. Math. 53 (1984) 125.

3. R. Kedem, T.R. Klassen, B. M. McCoy and E. Melzer, Phys. Lett. B304 (1993) 263.

4. E. Melzer, Int. J. Mod. Phys. A9 (1994) 133.

5. G. Felder, Nucl. Phys. B317 (1989) 215

6. G.E. Andrews, The theory of partitions, Cambridge Univ. Press (1984).

7. P. Di Francesco, P. Mathieu and D. Sénéchal, Conformal Field theory, Springer Verlag, 1997.

8. V. Kac, Modular invariance in mathematics and physics, address at the centennial of the AMS, (1988).

9. P. Christie, Int. J. Mod. Phys. A6 (1991) 5271.

10. J.J. Sakurai, Modern quantum mechanics, Addison Wesley (1994) sect. 3.8.

11. J. Lepowsky and R.L. Wilson, Inv. Math. 77 (1984) 199.

12. A.B. Zamolodchikov and V.A. Fateev, Sov. Phys. JETP 43 (1985) 215. 
13. J. Lepowsky and M. Primc, Contemporary Mathematics 46 AMS, Providence, 1985.

14. D. Gepner, Nucl. Phys. B290 (1987) 10.

15. B.L. Feigin, T. Nakanishi and H. Ooguri, Int. J. Mod. Phys. A7 Suppl. 1A (1992) 217.

16. T.R. Klassen and E. Melzer, Nucl. Phys. B338 (1990) 485; B370 (1992) 511.

17. V.V. Bazhanov and N. Yu Reshetikhin, Int. J. Mod. Phys. A4 (1989) 115; A. N. Kirillov, J. Sov. Math. 47 (1989) 2450; A. N. Kirillov and N. Yu Reshetikhin, J. Sov. Math. 52 (1989) 3156.

18. A. Klümper and P. A Pearce, J. Stat. Phys. 64 (1991) 13.

19. A.N. Kirillov, Dilogarithm identities, Prog. Theo Phys. Suppl. 118 (1995) 61-142.

20. W. Nahm, A. Recknagel and M. Terhoeven, Mod. Phys. Lett. A8 (1993) 1835.

21. M. Terhoeven, Lift of dilogarithm to partition identities, hep-th/9211120.

22. G.E. Andrews, Proc. Nat Acad. Sci. USA 71 (1974) 4082.

23. B. Gordon, Amer. J. Math. 83 (1961) 393.

24. A. Kuniba, N. Nakanishi and J. Suzuki, Mod. Phys. Lett. A 8 (1993) 1649.

25. A. Kuniba and N. Nakanashi, Mod. Phys. Lett. A 7 (1992) 3487.

26. R. Kedem and B. McCoy, J. Stat. Phys 71 (1993) 883.

27. S. Dasmahapatra, R. Kedem, B. M. McCoy and E. Melzer, J. Stat. Phys 74 (1994) 239.

28. R. Kedem, T.R. Klassen, B. M. McCoy and E. Melzer, Phys. Lett. B307 (1993) 68.

29. S. Dasmahapatra, R. Kedem, T.R. Klassen, B. M. McCoy and E. Melzer, Int. J. Mod. Phys. B7 (1993) 3617.

30. R. Kedem, B. M. McCoy and E. Melzer, in Recent progress in statistical mechanics and quantum field theory, ed. by P. Bouwknegt et al, World Scientific, (1995) 195.

31. G.E. Andrews, R.J. Baxter and P.J. Forrester, J. Stat. Phys. 35 (1984) 193.

32. A. Berkovich, Nucl. Phys. B415 (1994) 691.

33. A. Berkovich and B.M. McCoy, in Statistical physics at the eve of the 21st century, Series on Adv. in Stat. Mech., vol 14, ed. by M.T Batchelor and L.T. White, World Scientific, 1999.

34. F.D.M. Haldane, Phys. Rev. Lett. 67 (1991) 937.

35. F.D.M. Haldane, Phys. Rev. Lett. 60 (1988) 635; B.S. Shastry, Phys. Rev. Lett. 60 (1988) 639.

36. F.D.M. Haldane, Z.N.C. Ha, J.C. Talstra, D. Bernard and V. Pasquier, Phys. Rev. Lett. 69 (1992) 2021.

37. D. Bernard, V. Pasquier and D. Serban, Nucl. Phys. B 428 (1994) 612. 
38. P. Bouwknegt. A.A. Ludwig and K. Schoutens, Phys. Lett. B 338 (1994) 448; B 359 (1995) 304.

39. K. Schoutens, Phys. Rev. Lett. 79 (1997) 2608.

40. P. Bouwknegt. and K. Schoutens, Nucl. Phys. B 547 (1999) 501.

41. P. Jacob and P. Mathieu, Nucl. Phys. B 587 (2000) 514.

42. J. Distler and Z. Qiu, Nucl.Phys. B336 (1990) 533.

43. D. Nemeschansky, Nucl. Phys. B363 (1989) 665.

44. A.B. Zamolodchikov and V.A. Fateev, Sov. Phys. JETP 63 (1986) 913.

45. D. Gepner and Z. Qiu, Nucl. Phys. B285 [FS19] (1987) 423.

46. P. MacMahon, Combinatory analysis, 2 vols $(1917,1918)$, 3rd ed. reprinted by Chelsea, 1984.

47. G.E. Andrews, Houston J. Math. 7 (1981) 11.

48. V.A. Fateev and A.B.Zamolodchikov, Phys. Lett. 92A (1982) 37.

49. V.A. Fateev, Int J. Mod Phys. A6 (1991) 2109.

50. J. Lepowsky and R.L. Wilson, Proc. Nat. Acad. Sci. USA 78 (1981) 7254.

51. C. Dong and J. Lepowsky Generalized vertex algebras and relative vertex operators, Birkhaüser, 1993.

52. P. Jacob and P. Mathieu, Parafermionic Jacobi identities, Sugawara construction and generalized commutation relations, in preparation.

53. L. Bégin, C. Cummins and P. Mathieu, J. Math. Phys. 41 (2000) 7640. 